\title{
Miniature in vivo Robots for Remote and Harsh Environments
}

\author{
Mark E. Rentschler, Stephen R. Platt, Kyle Berg, Jason Dumpert, Dmitry Oleynikov, and Shane M. Farritor
}

\begin{abstract}
Long-term human space exploration will require contingencies for emergency medical procedures including some capability to perform surgery. The ability to perform minimally invasive surgery (MIS) would be an important capability. The use of small incisions reduces surgical risk, but also eliminates the ability of the surgeon to view and touch the surgical environment directly. Robotic surgery, or telerobotic surgery, may provide emergency surgical care in remote or harsh environments such as space flight, or extremely forward environments such as battlefields. However, because current surgical robots are large and require extensive support personnel, their implementation has remained limited in forward environments, and they would be difficult, or impossible, to use in space flight or on battlefields. This paper presents experimental analysis of miniature fixed-base and mobile in vivo robots to support MIS surgery in remote and harsh environments. The objective is to develop wireless imaging and task-assisting robots that can be placed inside the abdominal cavity during surgery. Such robots will provide surgical task assistance and enable an on-site or remote surgeon to view the surgical environment from multiple angles. This approach is applicable to long-duration space flight, battlefield situations, and for traditional medical centers and other remote surgical locations.
\end{abstract}

Index Terms-Extreme and remote environments, in vivo, robots, surgical, telementoring.

\section{INTRODUCTION}

$\mathbf{R}$ OBOT-ASSISTED surgery continues to advance laparoscopic surgery. Robots such as the da Vinci surgical system are currently being used to assist surgeons during minimally invasive surgery (MIS). They have significant advantages over manual laparoscopy, such as their ability to precisely control instruments, reduce hand tremor, and enable telesurgery. However, these robots are very large, expensive, and are currently limited to use in large surgical centers. A new area of surgical robotics focuses on placing robots entirely inside the patient. These in vivo robots currently lack some of the precise control provided

Manuscript received November 14, 2006; revised February 21, 2007.

M. E. Rentschler is with the University of Nebraska Medical Center, Omaha, NE 68198 USA (e-mail: mrentschler@unmc.edu).

S. R. Platt and K. Berg are with the Department of Mechanical Engineering, University of Nebraska, Lincoln, NE 68588 USA (e-mail: srp@unlserve. unl.edu; kyleaberg@gmail.com).

J. Dumpert is with the Department of Biomedical Engineering, University of Nebraska, Lincoln, NE 68588 USA (e-mail: jdumper1@bigred.unl.edu).

D. Oleynikov is with the Department of Surgery, University of Nebraska Medical Center, Omaha, NE 68198 USA, and also with the University of Nebraska, Lincoln, NE 68588 USA (e-mail: doleynik@unmc.edu).

S. M. Farritor is with the Department of Mechanical Engineering, University of Nebraska, Lincoln, NE 68588 USA, and also with the University of Nebraska Medical Center, Omaha, NE 68198 USA (e-mail: sfarritor@unl.edu).

Color versions of one or more of the figures in this paper are available online at http://ieeexplore.ieee.org.

Digital Object Identifier 10.1109/TITB.2007.898017 by systems such as the da Vinci. However, they have been shown to be useful in providing vision and task assistance [1], [2]. In addition, in vivo robots are small, inexpensive, and easily transported, making it more likely that this technology can be more widely adopted.

The Canadian Center for Minimal Access Surgery (CMAS) has done much research in the area of telerobotic surgery [3]. These efforts have included several missions with the National Aeronautics and Space Administration (NASA) using the Aquarius underwater habitat to test telerobotic surgery in remote and extreme environments. Aquarius, operated for the National Oceanic and Atmospheric Administration (NOAA) by the National Undersea Research Center (NURC) at the University of North Carolina Wilmington (UNCW), is a submersible habitat that houses crews 20-m underwater for up to several weeks off the coast of Key Largo, FL. The NASA Extreme Environment Mission Operations (NEEMO) uses Aquarius several times each year to provide analogous extravehicular activity (EVA) and extended duration training for Astronauts.

CMAS had teamed with the NASA during NEEMO 7, October 2004, and NEEMO 9, April 2006 to perform surgical training experiments. During NEEMO 9, in vivo robots from the University of Nebraska were also tested and evaluated by the crew. All four members of the crew performed simulated surgical tasks that included bowel inspection, stretch and dissect, and appendectomy procedures. The surgical environment was replicated by placing synthetic materials inside an abdominal cavity simulator, as shown in Fig. 1. The Aquanauts were trained before the mission to perform the bowel inspection and stretch and dissect procedures using both a traditional laparoscope and a fixed-base tilting camera robot. The crew received no training for the appendectomy; this task required that the crew apply the skills developed during the bowel inspection and stretch and dissect procedures while being telementored through the appendectomy by the University of Nebraska team, remotely located in Omaha, NE.

This paper describes our current work to develop small and easily transportable in vivo robots for use in extreme and remote environments, such as long-duration space flight and battlefield situations. The purpose of the miniature in vivo surgical robots tests presented here was to evaluate the ease of use and the time required to perform simple laparoscopic tasks utilizing different vision systems (laparoscope, and in vivo camera robots). The ability to use these robots as part of a telementoring system to guide crew members through an unfamiliar surgical procedure (appendectomy) in a remote harsh environment was also assessed. 

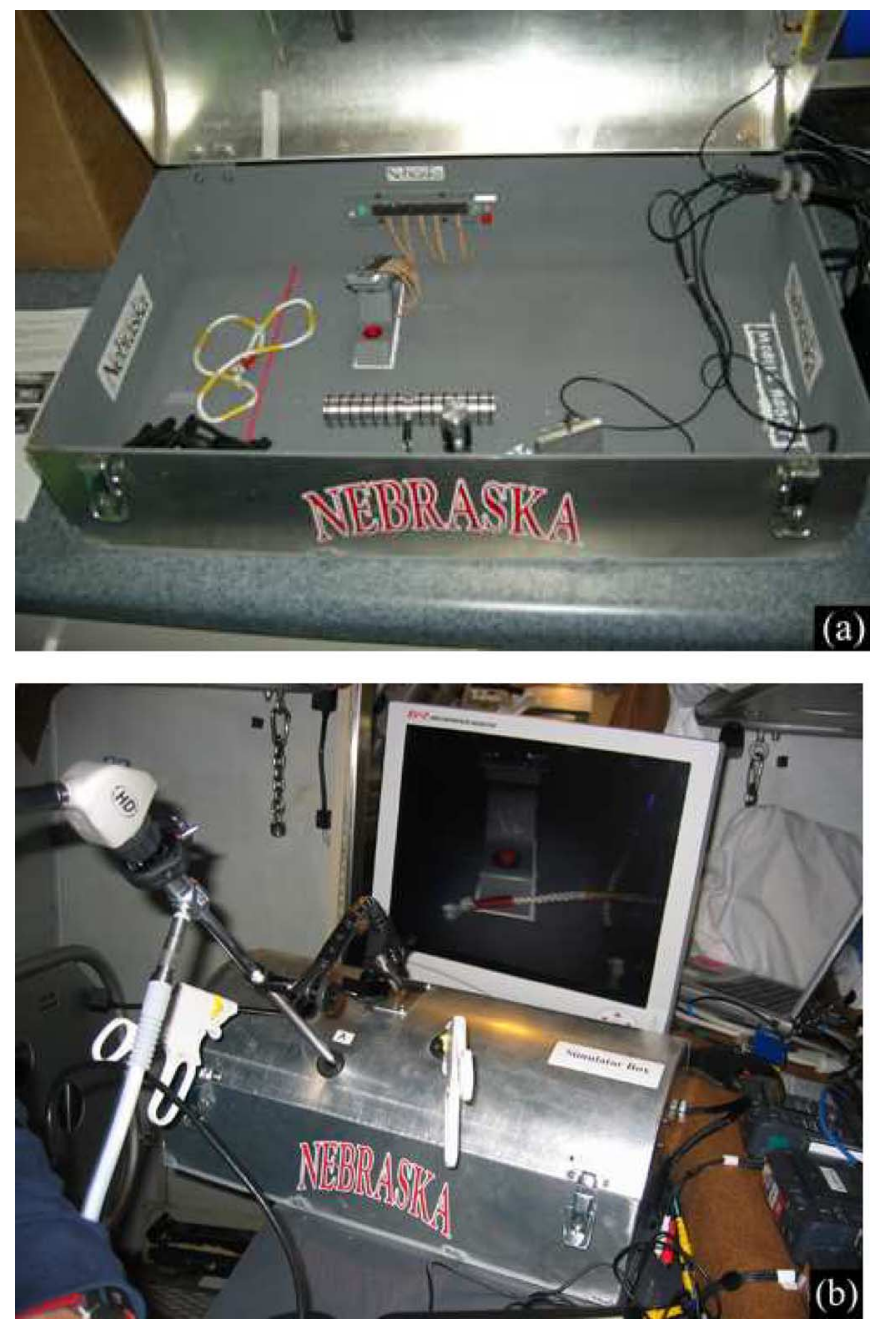

Fig. 1. (a) Abdominal cavity simulator equipment setup and (b) prepared for task completion in the Aquarius habitat.

\section{BACKGROUND}

\section{A. Robot-Assisted Surgery}

The use of robots is currently recognized as a major driving force for advancing MIS [4]-[6]. However, current surgical robots, such as the da Vinci surgical system made by Intuitive Surgical, have several significant limitations. Although one recent report concluded that robotic surgery can enhance dexterity compared to traditional laparoscopy [7], most studies suggest that current robotic systems offer little or no improvement over standard laparoscopic instruments in the performance of basic skills [8]-[10]. Current systems also remain constrained by limited sensory and mobility capabilities, and their high cost and extensive support requirements make them unavailable to most hospitals.

\section{B. Other in vivo Robots}

While much effort has focused on surgical robots in the operating room, recent research also focuses on placing robots completely (or mostly) inside the patient. These in vivo robots help to overcome the constraining limitations of working through small incisions. The simplest such mechanisms have been endoscopes that include actuators that can turn the endoscope tip after it enters the body [11], [12], leaving the power and control equipment outside the body. Other scopes developed to explore hollow cavities such as the colon or esophagus have included locomotion systems based on "inch-worm" motion that use a series of grippers and extensors [13], [14], rolling tracks [15], or rolling stents [16]. Another approach for exploring the gastrointestinal (GI) tract is swallowing an un-tethered camera pill. One such commercially available device, called M2 A from Given Imaging Ltd [17], [18], returns multiple (thousands) images as it naturally moves through the GI tract. However, because the device is entirely passive, it cannot be directed to image a particular location and the exact locations of the images are not known. Combined with the very large volume of images, the use of this device for diagnosis is difficult. Other work has focused on a mobile robot to traverse the surface of a beating heart [19], [20]. This robot uses suction cups and has demonstrated successful prehension, turning, locomotion, and dye injection in a porcine (pig) model.

Most of the in vivo robots described earlier are designed to function in very specific locations in the body. They are all either nonmobile, or their mobility systems require narrow hollow cavities, external power, or natural processes to function. The in vivo robots used for this study have been developed for use in the open environment of an insufflated abdomen during laparoscopic surgery.

The in vivo robots presented here have CMOS camera imagers onboard that provide video feedback to the surgical team. As low-cost CMOS technology continues to improve, so will the video quality of these robots. Two recent studies were conducted to determine efficacy of in vivo camera robots versus a standard laparoscopic camera [21] using simulated surgical tasks and color/resolution charts [22]. Results showed no significant difference in performance for the two vision systems.

\section{Telementoring and Telerobotic Surgery}

The earliest use of telemedicine can be traced back to 1959 , when doctors used two-way video communication systems for psychiatric consultation in Nebraska [23]. This innovative pioneering project helped lead the way to present telementoring and telesurgery possibilities. Surgical telementoring has now been used for over a decade for both training young surgeons in routine endoscopic surgery and advanced operations [24]. Such telementoring has been shown to be as effective for development of surgical skills as local mentoring when video and audio quality are sufficient [25]. In fact, telemedicine in today's world of video conferencing includes education, training, consulting, and mentoring. Assessment results of telemedicine in surgical education and patient care show improved diagnostic potential with accurate telediagnoses for surgical cases of 95\% [26].

Early efforts at telerobotic surgery focused on developing the robot kinematics and trajectory planning for telelaparoscopic manipulation [27]. This included development of robotic extenders that could be indirectly controlled by the surgeon through a master arm. This master-slave robot system controls the 
movement of the robot extender inside the abdominal cavity, which is controlled indirectly by hand movements of the surgeon on a telesurgical workstation. The other major research focus was the development of a robust, fail-safe, and resilient real-time communications system [28] and determination of acceptable time delays that did not grossly affect surgical accuracy and dexterity [29]. These research efforts were combined in the early part of the 21st century when the Zeus surgical system received Food and Drug Administration (FDA) clearance and its use in medical centers began. Early results showed that computeraided surgery (CAS) was safe and feasible, with patient recovery times similar to those of conventional surgery [30]. Another major advance in the CAS occurred in September 2001, when the first example of transatlantic telesurgery was demonstrated with a patient in Strasbourg and a surgeon in New York [31]. This showed that computer technology, robotics, fiber optics, and surgical techniques had advanced sufficiently to overcome the problems that had previously prevented successful longdistance telesurgery, such as time delays, tremor filtering, and insufficient visual feedback. Today, telerobotic surgery is routinely being used to provide high-quality laparoscopic surgical services to patients in remote regions of Canada using a commercially available network with $15 \mathrm{Mb} / \mathrm{s}$ of bandwidth [32].

To be successful, in vivo telerobots will need to be provided with enough power, sufficient in vivo illumination, and largeenough bandwidth to provide visual feedback to the remote surgical team. The power requirement for the robots described here is approximately $300 \mathrm{~mA}$ at $3 \mathrm{~V}$. Commercially available lithium oxide batteries have been successful in powering these in vivo robots for up to 50 minutes. The Ultrabright LEDs used with the camera robots each produce $10000 \mathrm{mcd}$, which has provided sufficient illumination. The CMOS imagers provide video output in the National Television System Commission (NTSC) format at 30 frames/s. We have used analog transmission thus far to maintain a lower bandwidth requirement. However, moving to telerobotic surgery with in vivo robots will likely require digital transmission. For digitally transmitted video quality comparable to video conferencing the necessary bandwidth range is $10-15 \mathrm{Mb} / \mathrm{s}$.

Telerobotic surgery has generally been performed in traditional medical centers equipped with systems such as da Vinci. However, the size and expense of this equipment limits telesurgery's presence in remote and harsh environments. Providing inexpensive in vivo robots that can be remotely controlled would provide telesurgical access and telementoring in these remote locations (small rural hospitals, long-duration space flight), and harsh environments (forward battlefield locations). In locations where telesurgical equipment exists, such in vivo robots could be used to help augment the surgical view and provide surgical assistance.

\section{NASA EXTREME ENGINEERING MisSION OPERATIONS}

\section{A. Habitat Description}

Aquarius, shown in Fig. 2, is an underwater ocean laboratory located in the Florida Keys National Marine Sanctuary. The 73.5-t laboratory $(13 \mathrm{~m} \times 6 \mathrm{~m} \times 5 \mathrm{~m})$ is deployed three

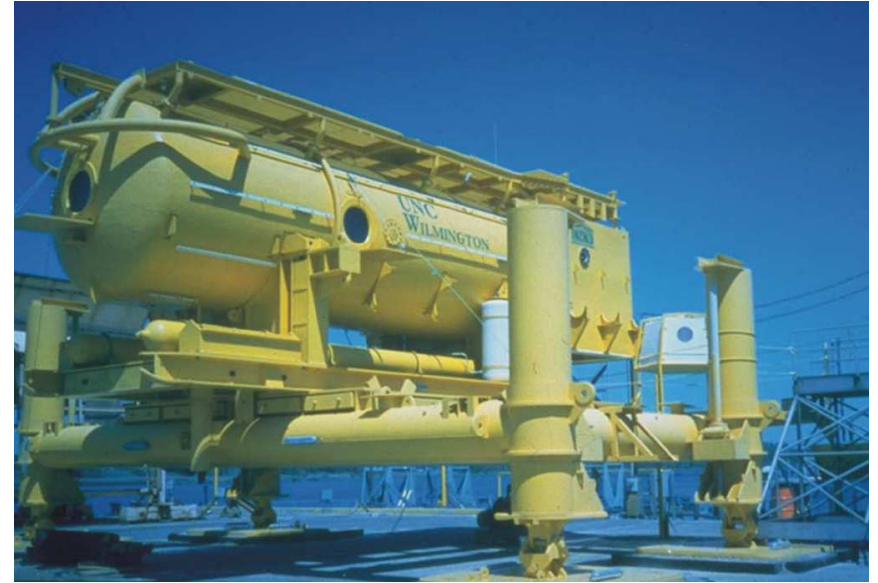

Fig. 2. Aquarius habitat on the dock during maintenance (Image provided courtesy of the NOAA's Undersea Research Center at the University of North Carolina, Wilmington).

and half miles offshore, at a depth of $20 \mathrm{~m}$. Aquarius houses six bunks, a restroom, and kitchen. Aquarius is an ambient pressure habitat, with its interior atmospheric pressure equal to the surrounding water pressure. Scientists live in Aquarius during multiple weeklong missions using saturation diving to study and explore the coastal ocean. Astronauts use Aquarius for extended training for space flight. Aquarius is owned by the NOAA and is operated by the NOAA Undersea Research Program's (NURP) Undersea Research Center at the University of North Carolina, Wilmington.

\section{B. Mission Description}

The NEEMO 9 mission was a joint project involving the CMAS at McMaster University, the University of Nebraska, the U.S. Army Telemedicine and Advanced Technology Research Center (TATRC), the National Space Biomedical Research Institute (NSBRI), and the NASA. The mission built upon the success of the NEEMO 7 mission and continued to evaluate new medical diagnostic and therapeutic technologies to enhance the delivery of state-of-the-art medical care in remote and harsh environments. Another goal of the mission was to develop procedures and techniques for lunar exploration using remotely operated vehicles, tracking systems, and navigation devices. The Aquanaut crew included three NASA Astronauts trained in engineering and general surgery, and one advanced laparoscopic surgeon.

Each member of the four-person crew completed the tasks onboard Aquarius, as shown in Fig. 3. These results helped to validate in vivo camera robots as an effective alternative to laparoscope use. The telementoring results demonstrated that nonsurgeons having been trained with a specified skill set can be telementored to build on that skill set and perform a more complex laparoscopic procedure using in vivo robots.

\section{In vivo RоBOTs}

Much effort has been spent developing in vivo robots for vision and task assistance during endoscopic surgery. The robots function in the insufflated abdominal cavity, which is below the 

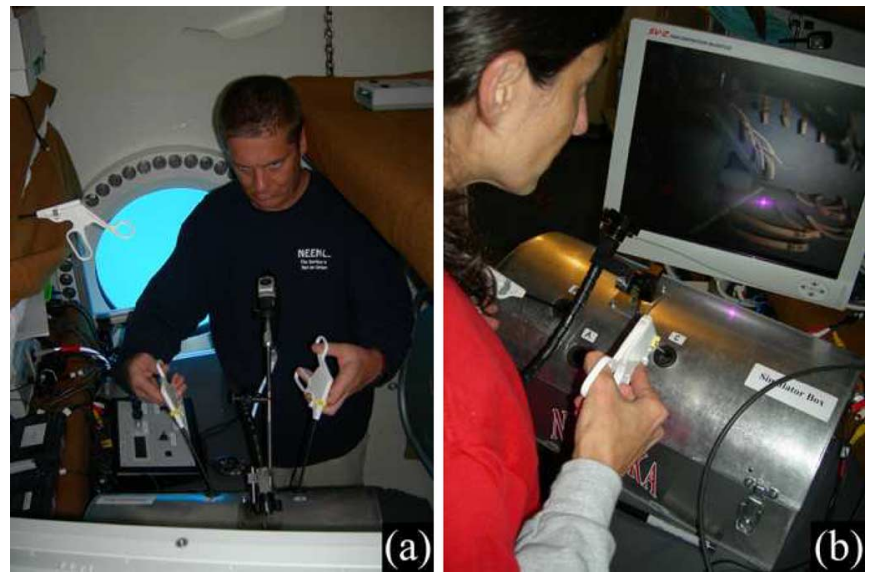

Fig. 3. Crew members completing miniature in vivo robot surgical tasks onboard Aquarius (Images provided courtesy of the NASA).

diaphragm. Breathing does create a shallow rise in the surgical field, but has not drastically affected the performance of the robots. We continue to strive to reduce the size of our robots and are currently developing most of our robots in the $12-15-\mathrm{mm}$ diameter range and 50-75-mm length range. The abdominal cavity is generally $20 \mathrm{~cm} \times 15 \mathrm{~cm}$ and is $8 \mathrm{~cm}$ in height when fully insufflated. This is the "working" space for the robotic team. The robots need to fit through a rigid trocar port (typical sizes are $12-15 \mathrm{~mm}$ in diameter). Two broad classes of in vivo robots have emerged: fixed base and mobile. The fixed-base camera robots, similar to the tilt camera robot used during the NEEMO 9 mission [Fig. 4(a)], can provide visual feedback to the surgeon. These robots have adjustable-focus capability and usually several DOFs that allow the view from the robot to be panned and tilted. Several designs have incorporated LEDs. Most of these devices have been tethered for power and video feedback, although wireless versions have also been tested with success. Several different attachment mechanisms have been used for these robots. A spring-loaded foldable-tripod platform was used for the NEEMO 9 test. This 1-DOF tilting robot is $15 \mathrm{~mm}$ in diameter (when the legs are abducted during insertion and retraction) and $60-\mathrm{mm}$ tall. A permanent magnet dc motor is used to actuate the tilting mechanism and is controlled by the crew member through a switch. The folding tripod base allows the robot to be easily inserted into and removed from the abdominal cavity through a standard trocar port.

This type of robot has previously been used successfully in animal tests, during which the visual feedback from the robot was used exclusively to perform a canine prostatectomy (prostate removal) and nephrectomy (kidney removal) [33]. The tilting camera robot shown in Fig. 4(a) was used by the NEEMO 9 crew during the bowel inspection and stretch and dissect tasks, as discussed in Section V. Mobile in vivo robots were developed to provide a mobile platform for visual feedback and task assistance. These robots have two independent motors that separately drive each wheel, which provides forward, reverse, and turning capabilities for the two-wheeled mobile platform, shown in Fig. 4(b). A wheel profile was developed such that these robots can traverse abdominal organs without causing tis-
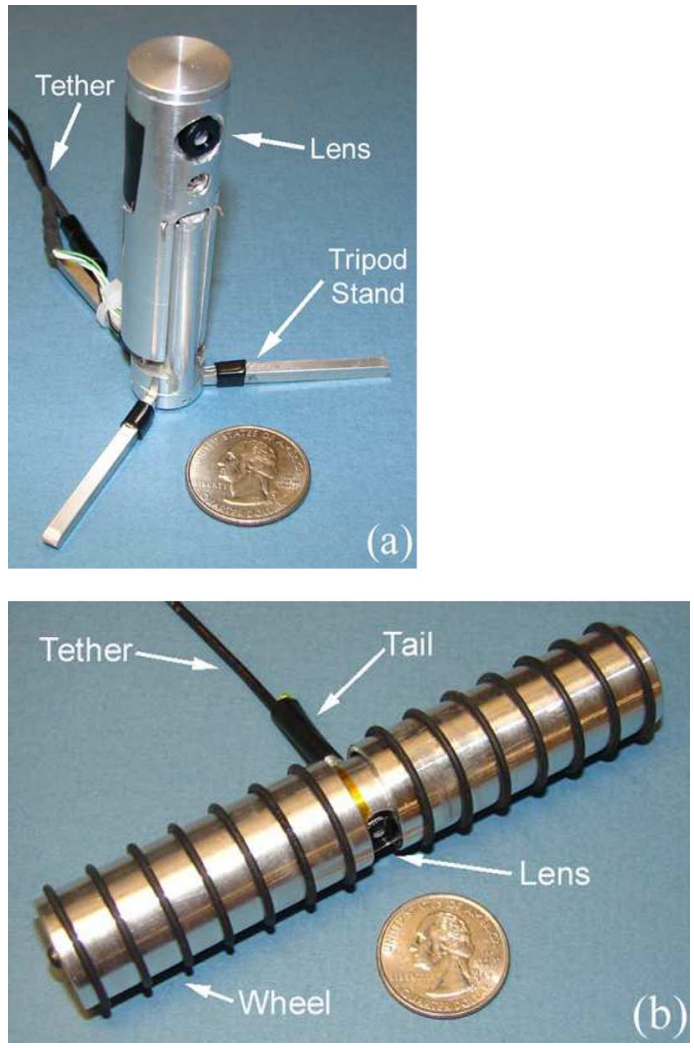

Fig. 4. (a) Tilting camera and (b) mobile camera in vivo robots used during the NEEMO 9 mission.

sue damage [34]. The wheel profile used in the current work is much different due to the nature of the experiments performed during NEEMO 9. The mobile camera robot shown in Fig. 4(b) is $110 \mathrm{~mm}$ in length and $20 \mathrm{~mm}$ in diameter. This robot is controlled by the crew member using a joystick. Both of the NEEMO 9 robots also have onboard adjustable-focus cameras that provide visual feedback. The focusing mechanism is driven by a motor and a series of gears that change the position of the imager relative to the lens. A mobile camera robot, similar to the one shown in Fig. 4(b) has been used to provide exclusive visual feedback to a surgeon during a porcine cholecystectomy (gallbladder removal) [1]. A mobile camera and biopsy robot were also developed and used successfully to biopsy portions of hepatic (liver) tissue during an animal surgery [2]. Such robots have also recently been used to demonstrate the feasibility of performing natural orifice transgastric endoscopic surgery (NOTES), wherein a robot is introduced to the abdominal cavity through an incision in the gastric cavity instead of the traditional approach of keyhole surgery with trocars placed in the abdominal wall [35]. The mobile camera robot shown in Fig. 4(b) was used by the NEEMO 9 crew to provide visual feedback during the appendectomy procedure, as discussed in Section V.

\section{NEEMO 9 TASKS}

The crew performed the surgical tasks using the abdominal cavity simulator shown in Fig. 1. During the tasks, the crew manipulated the tissue specimens using laparoscopic tools 

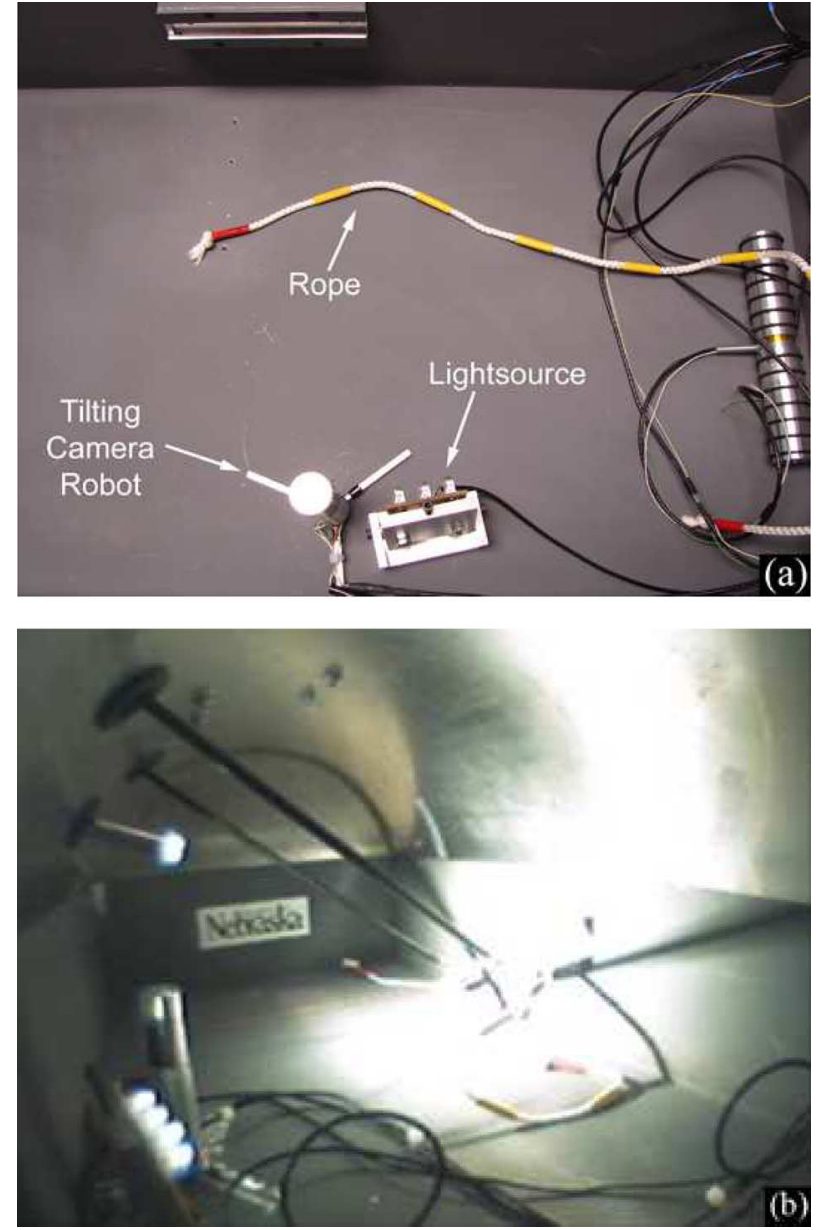

Fig. 5. (a) Overview of the robot and rope position setup for the rope passing task and (b) the abdominal cavity simulator camera view of the rope passing task using the laparoscope's video feedback.

inserted through the trocar holes in the cover of the simulator. The crew observed their performance by watching the video feedback from the in vivo robots or laparoscope on a computer screen, as shown in Fig. 3. The same screen was used for all video feedback and the position of the computer screen was unchanged for each set of tasks.

\section{A. Bowel Inspection/Rope Passing}

The bowel inspection task involved passing a rope with colored segments from laparoscopic grasper to laparoscopic grasper while only contacting the colored portions, spaced every $5 \mathrm{~cm}$. This task replicates the surgical task used to inspect very long sections of bowel. The rope was passed back and forth five times using the handheld laparoscopic graspers. Each crew member performed this task one time using a 0-degree laparoscope and one time using the tilting camera robot for visual feedback. The rope was initially positioned as shown in Fig. 5(a). The tilting camera robot and light source were positioned over fixed location markings on the simulator box bottom, as shown in Fig. 5(a), and the laparoscope was positioned above the tilting camera robot, as shown in Fig. 5(b). These two positions are representative of the nominal positions used during
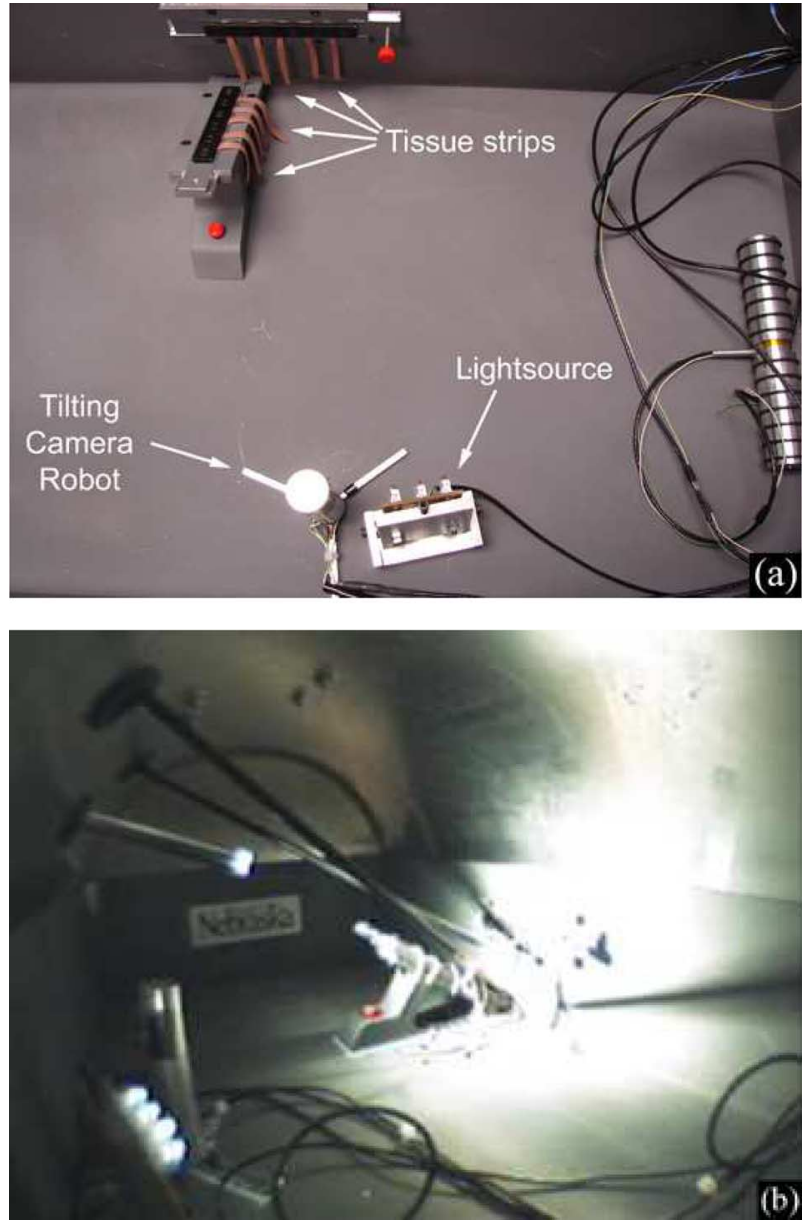

Fig. 6. (a) Overview of the robot and specimen setup for the stretch and dissect task and (b) the abdominal cavity simulator camera view of the stretch and dissect task using the laparoscope's video feedback.

laparoscopic surgery. The tilting camera robot used a separate light source and the imager settings were adjusted to match this illumination. The laparoscope used its integrated xenon light source for illumination.

\section{B. Stretch and Dissect}

The stretch and dissect task involved grasping synthetic strips of tissue and cutting down a marked line on the tissue using laparoscopic tools. This task simulates surgical laparoscopic dissection. Each crew member also performed this task one time using the laparoscope and one time using the tilt camera robot for visual feedback. The same lighting and positioning of tools, robots, and light sources were used for the stretch and dissect and the bowel passing tasks.

Two sets of tissue strips were placed, as shown in Fig. 6(a). One set of five strips was placed on an incline so that the crew member would need to pull the tissue to the right to cut along the marked location. The other set of five strips was placed along the back wall of the simulator so that the crew member would need to pull the tissue down to cut along the marked location. 

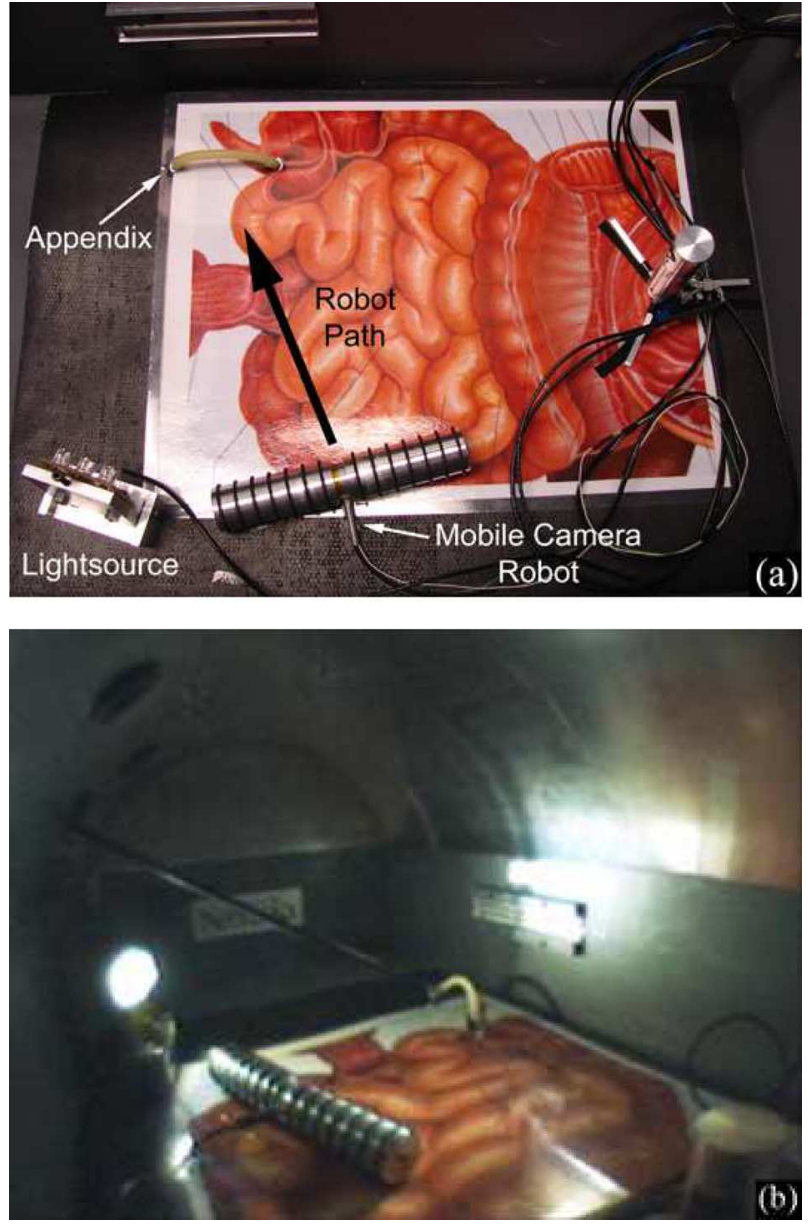

Fig. 7. (a) Overview of the robot and appendix position setup for the appendectomy task and (b) the abdominal cavity simulator camera view of the appendectomy task using the mobile robot's video feedback.

\section{Appendectomy}

This task was completed without specific previous training to evaluate whether a skill set can be built upon and the crew members could be telementored through an unfamiliar surgical procedure using the in vivo robots. The crew had been trained beforehand on use of the surgical tools, including control of the mobile camera robot. Immediately prior to starting this task, each crew member watched a short 30-s video describing the appendectomy procedure. The crew member was then telementored, using video conferencing software, about the nuances of the procedure and how best to complete the task. The mobile camera robot and light source were positioned over markings on the simulator box bottom, as shown in Fig. 7(a). Finally, the crew member performed the task while being telementored. The appendectomy procedure involved driving the mobile camera robot toward an appendix (as shown in Fig. 7) that was similar in size and anatomy to a patient, using only video feedback from the robot. After positioning the mobile robot to optimize the view, the crew member performed the appendectomy by stapling, dissecting, and removing the appendix.

The most common complication associated with an appendectomy is infection of the wound (surgical incision). The sec-
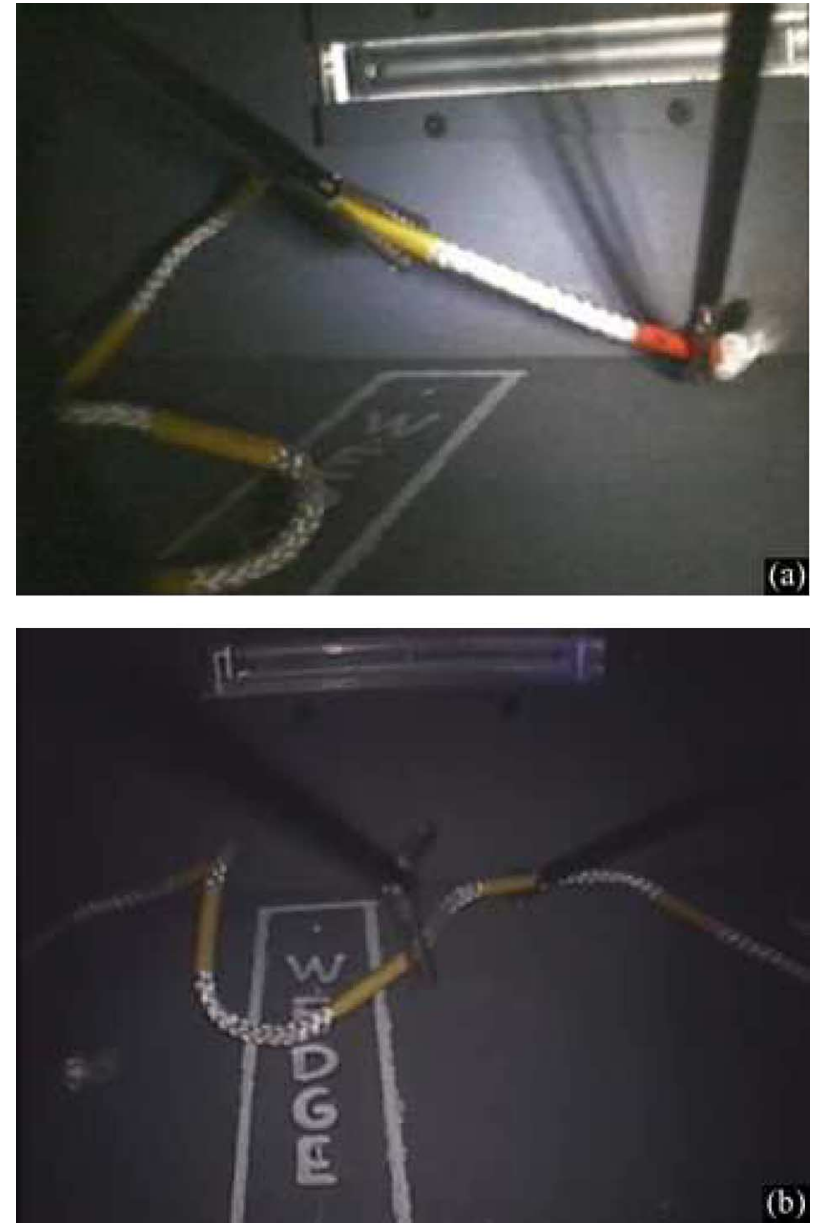

Fig. 8. (a) View from the tilting camera robot and (b) laparoscope during the rope passing task.

ond most common complication is perforation of the appendix leading to abdominal infection. Both complications are usually treated with antibiotics. For future studies, we may create a simulation appendix with fluid inside the appendix to examine if the procedure can be completed while preventing any leakage.

\section{EXPERIMENTAL RESULTS}

The video recorded during each task for each crew member was reviewed after the mission. Raw data were then compiled using this video to evaluate performance metrics.

\section{A. Rope Passing}

The rope passing task was individually and successfully completed by each crew member during the mission using visual feedback from the tilting camera robot [Fig. 8(a)] and the laparoscope [Fig. 8(b)]. This task was timed and accuracy was determined by postprocessing the video data including only the time required to complete the task and excluding setup time.

For the rope passing task, the metrics used were time, number of hits, number of misses, and number of grasps. Hits were defined by the number of times the crew member correctly grasped the colored rope segments, misses were defined as 
TABLE I

RoPe PASSING DATA ANALYSIS

\begin{tabular}{cccccc}
\hline \hline Metric & $d f$ & $t$ & $p$ (two-tailed) & $\mu_{\text {robot }}$ & $\mu_{\text {laparoscope }}$ \\
\hline Hits & 3 & -0.792 & 0.486 & 97.25 & 100.25 \\
Misses & 3 & -2.202 & 0.115 & 11.00 & 17.75 \\
Grasps & 3 & -2.168 & 0.119 & 108.25 & 118.00 \\
Time (s) & 3 & -0.755 & 0.505 & 260.25 & 285.25 \\
\hline \hline
\end{tabular}

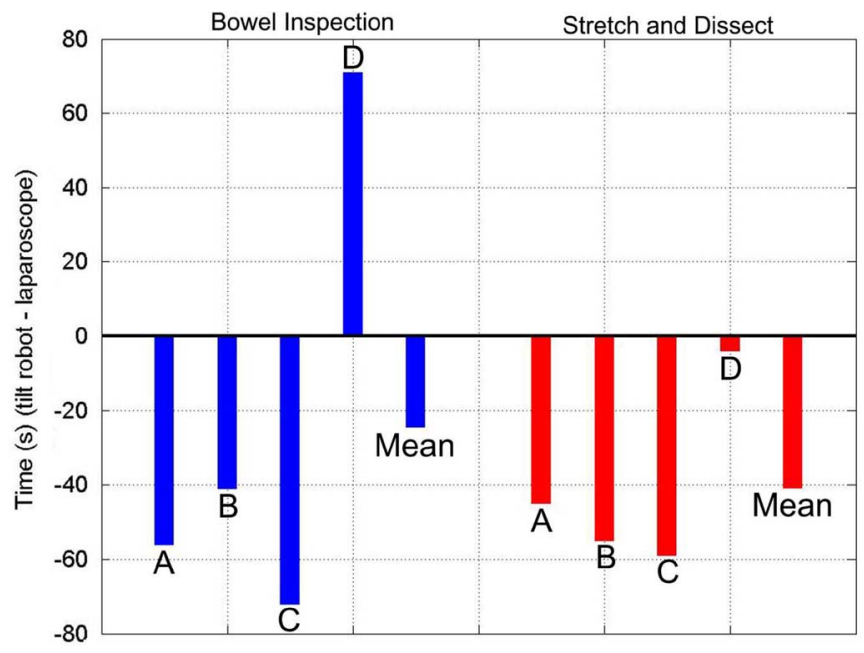

Fig. 9. Time-difference plots (tilt robot time minus laparoscope time) for each crew member for both the bowel inspection task and the stretch and dissect task.

the number of times they grasped the rope between the colored segments, and the number of grasps was the sum of hits and misses. Perfect completion of the task would be to touch only the colored segments while passing the rope back and forth five times, as previously described. This would result in 100 hits and no misses. During completion of this task, each crew member touched the rope (miss) and/or grabbed an individual colored segment (hit) more than one time each during the pass. Therefore, the results include performances where the number of hits exceeds 100 .

Paired $t$ tests were computed for each crew member, with the pairs of data corresponding to the visual feedback system used, either laparoscope or tilting camera robot. These data are summarized in Table I, where $d f$ is the degrees of freedom $(N-1), t$ is the $t$ statistic, $p$ is the 2-sided significance level, and $\mu$ is the group mean. Degrees of freedom ( $d f$ ), as used in statistical analysis, is a measure of the number of independent pieces of information on which the precision of a parameter estimate is based. In this case, $d f=4-1$ because we are using the data from the four crew members to calculate a mean for each metric. There was no significant difference $(p>0.05)$ between the two vision systems for each of the four metrics.

The plots shown in Fig. 9 summarize the differences in time required for each crew member to complete the bowel inspection and stretch and dissect tasks using each of the two vision systems. All but one crew member completed each of the two tasks more quickly using video feedback from the tilt robot compared to that from the laparoscope. The cause of crew member D's apparently anomalous result can only be subject to spec-
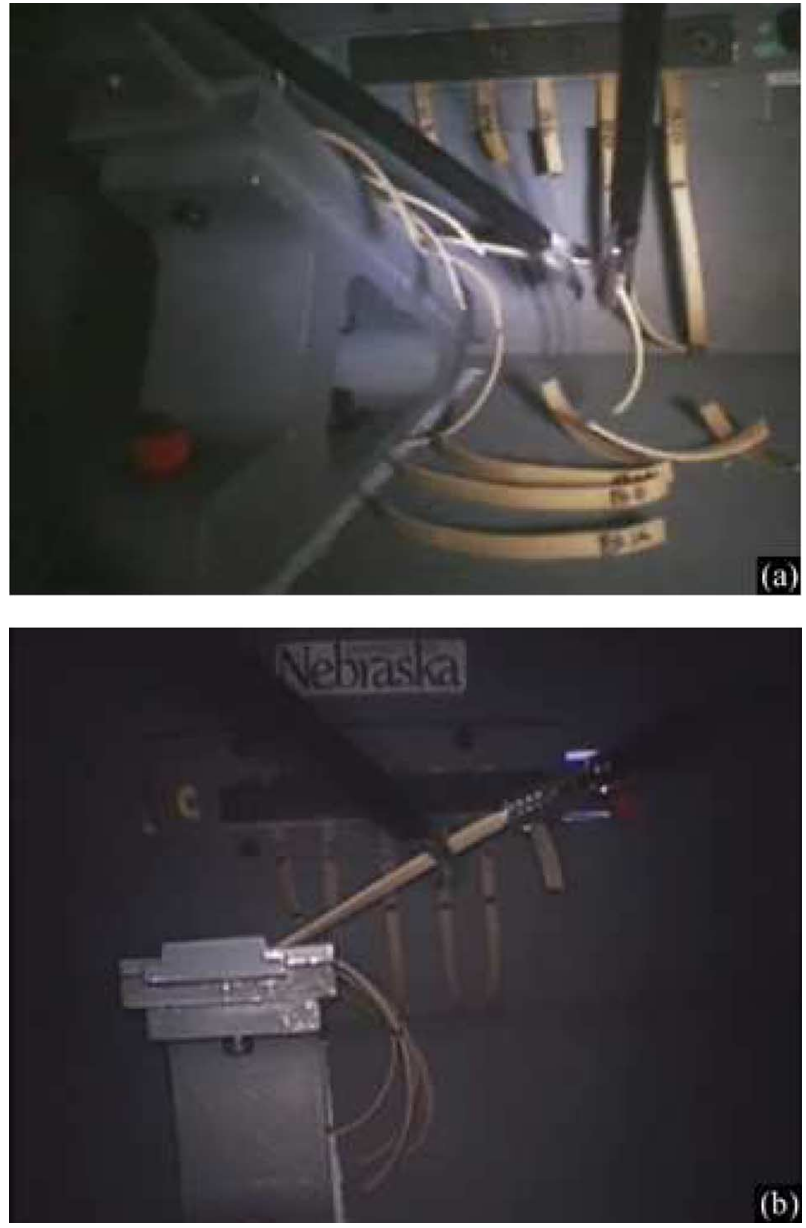

Fig. 10. (a) View from the tilting camera robot and (b) laparoscope during the stretch and dissect task.

ulation. However, if the timed data are analyzed without crew member $\mathrm{D}(N=3, d f=2)$, the $t$ statistic changes significantly $(t=-6.29)$ and the $p$ value decreases $(p=0.024)$, which indicates a significant difference. These results suggest that video feedback from the tilting camera robot is at least as good as that from the laparoscope for this task.

\section{B. Stretch and Dissect}

The stretch and dissect task was individually and successfully completed by each member of the crew during the mission using visual feedback from the tilting camera robot [Fig. 10(a)] and the laparoscope [Fig. 10(b)]. This task was timed and accuracy was determined by posttest measurements of the tissue specimens.

The metrics used for the stretch and dissect task were distance of cut, angle of cut, and time. The distance metric was defined to be the distance in millimeters between the indicated cutting position mark on the specimen and the actual cut position. The angle metric was the angular error between the actual cut and the indicated mark on each specimen, measured in degrees. The time metric was task duration in seconds. The absolute value of the error was used for both the distance and angle metrics. Paired $t$ tests were again used to analyze the data, and the results are summarized in Table II. 
TABLE II

STRETCH AND DISSECT DATA ANALYSIS

\begin{tabular}{cccccc}
\hline \hline Metric & $d f$ & $t$ & $\begin{array}{c}p \\
\text { (two-tailed) }\end{array}$ & $\mu_{\text {robot }}$ & $\mu_{\text {laparoscope }}$ \\
\hline Angle (deg) & 3 & -0.853 & 0.456 & 11.25 & 14.00 \\
Distance (mm) & 3 & 0.488 & 0.659 & 1.08 & 0.95 \\
Time (s) & 3 & -3.234 & 0.048 & 106.00 & 146.75 \\
\hline \hline
\end{tabular}
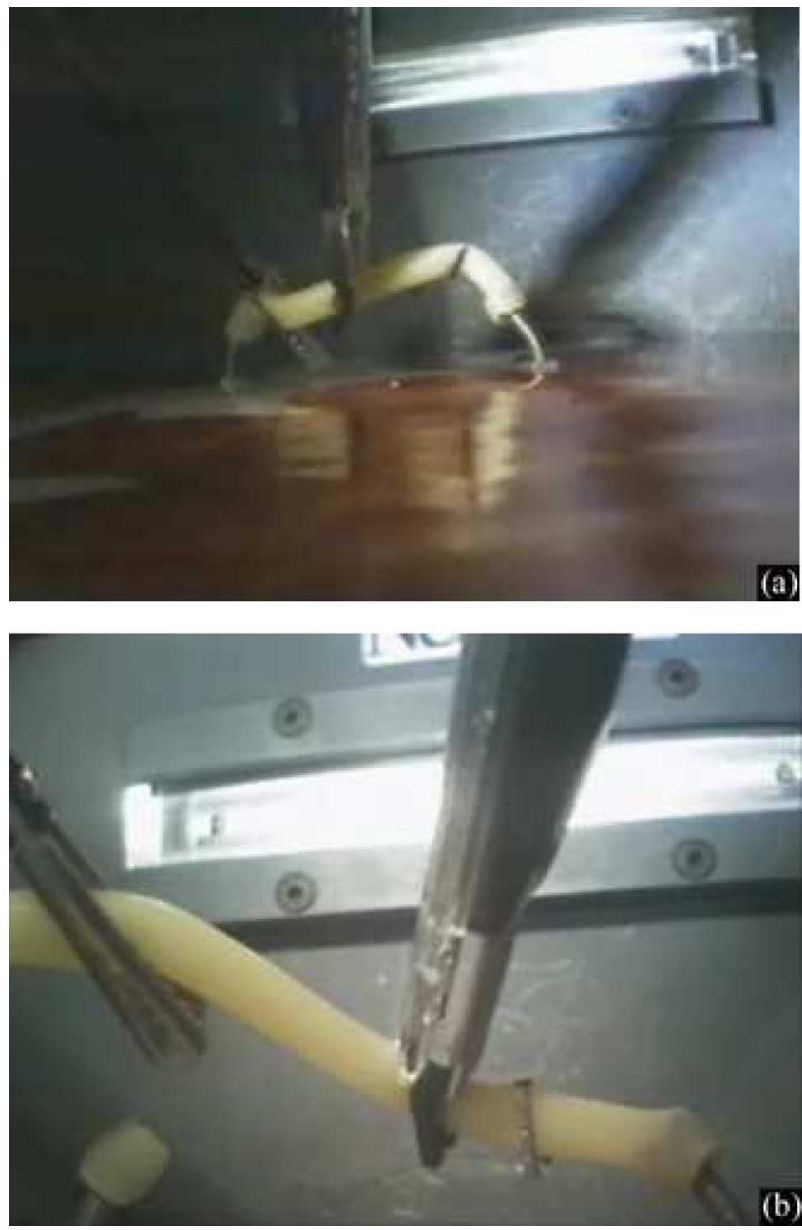

Fig. 11. (a) Distant view and (b) close-up view from the mobile camera robot during the appendectomy.

There is no significant difference $(p>0.05)$ between the two vision systems when comparing the angle and distance metrics. However, the time metric shows a significant difference $(p<0.05)$, with the mean time using the tilt robot $40.75 \mathrm{~s}$ less than the mean time for the laparoscope.

\section{Appendectomy}

Each member of the crew successfully completed the appendectomy task. Several views from the mobile camera robot are shown in Fig. 11. These results help demonstrate that the crew can be trained on a specific skill set and that through telementoring they can build upon this skill set to complete more complex tasks.
TABLE III

SURVEY RESULTS

\begin{tabular}{cccccc}
\hline \hline Category & $d f$ & $t$ & $\begin{array}{c}p \\
\text { (two-tailed) }\end{array}$ & $\mu_{\text {robot }}$ & $\mu_{\text {laparoscope }}$ \\
\hline $\begin{array}{c}\text { Depth } \\
\text { Perception }\end{array}$ & 2 & 0.000 & 1.000 & 4.00 & 4.00 \\
Lighting & 3 & -5.196 & 0.014 & 2.75 & 4.25 \\
Video Feedback & 3 & -1.567 & 0.215 & 3.50 & 4.25 \\
Camera Control & 3 & -0.243 & 0.814 & 3.25 & 3.50 \\
$\quad$ Overall & 3 & 0.000 & 1.000 & 3.50 & 3.50 \\
Functionality & & &
\end{tabular}

\section{Survey Results}

After completing the surgical tasks, each crew member was asked a series of survey questions related to depth perception, lighting, video feedback, camera control, and overall functionality of the camera robots and the laparoscope. Crew members answered the questions on a scale from 1 to 5 , with 5 being the best. Paired $t$ tests were used to analyze these data. The results are summarized in Table III.

In all categories, except for one, there was no significant difference $(p>0.05)$ in scores between the two systems. The survey results indicate that better lighting would be helpful when using the in vivo camera robots. To help augment this, current work has focused on adjusting onboard camera settings, such as brightness, hue, saturation, and color to better fit the low-lighting conditions during surgery in the abdominal cavity. Analysis of the survey answers indicates that the use of telementoring with the use of surgical robots could be beneficial.

\section{E. Results Summary}

The combined results from these tests show that there was no significant difference between the two vision systems when comparing task accuracy. However, there was a significant decrease in the time required to complete the tasks when using the in vivo camera robots. These results show that video feedback from the tilting camera robot is at least as good as, and perhaps superior to, that from the laparoscope for these tasks. The telementoring task results help demonstrate that individuals can be trained on a basic skill set and that through telementoring they can build upon this skill set to complete more complex procedures.

\section{FUTURE DIRECTIONS}

In vivo robots for endoscopic surgery have the potential to be used in traditional medical centers, remote surgical locations, extreme environments such as battlefields, and harsh environments such as long-duration space flight. The goal of the current research is to provide improved visual feedback to the surgeon through multiple in vivo video systems, provide in vivo tissue manipulation and task assistance, and reduce patient trauma by requiring only a single incision through which multiple robots could be inserted. Because these devices are very small and portable, their use in remote, harsh, and extreme environments will provide surgical assistance otherwise not possible. As the 
technology develops, improvements may include telecontrol, task assistance, and some autonomy.

\section{SUMMARY AND CONCLUSION}

This paper presents the results from the NEEMO 9 mission during which in vivo robots from the University of Nebraska were tested and evaluated by a four-member crew comprised of three NASA Astronauts and one surgeon. The crew performed simulated surgical tasks that included bowel inspection, stretch and dissect, and appendectomy procedures. The surgical environment was replicated by placing synthetic materials inside an abdominal cavity simulator, and the procedures were performed using standard laparoscopic tools in combination with video feedback from a traditional laparoscope or in vivo camera robots. The Aquanauts were trained before the mission to perform the bowel inspection and the stretch and dissect tasks. The crew was not pretrained to perform the appendectomy. The appendectomy task required the crew to use the in vivo camera robots and the skills developed in the other two tasks while being telementored via videoconferencing through the appendectomy by the University of Nebraska team at Omaha.

Although the sample size is small $(N=4)$, for both the rope passing and stretch and dissect tasks, there was no significant statistical difference in task accuracy between the two visual feedback systems. However, there is a significant decrease in the times required to complete the tasks when using the tilting camera robot. The appendectomy results show that the crew could build upon a core set of skills to perform a new procedure using in vivo robots and telementoring. The combined results from these tests suggest that the use of miniature surgical robots could be used in place of standard laparoscopic surgical equipment without loss of performance. These results show that video feedback from the in vivo camera robots is at least as good as that from the laparoscope for these tasks. The use of these robots can potentially reduce patient trauma in traditional medical centers, while the size of the robots makes them ideal for transportation to and use in remote or harsh environments.

\section{ACKNOWLEDGMENT}

The authors would like to thank the CMAS for leading this project and the NURC for the opportunity to conduct this exciting research. We would also like to thank everyone who helped topside with NEEMO 9, with a special thanks to the NEEMO 9 crew including the engineering support team.

\section{REFERENCES}

[1] M. Rentschler, J. Dumpert, S. Platt, S. Ahmed, S. Farritor, and D. Oleynikov, "Mobile in vivo camera robots provide sole visual feedback for abdominal exploration and cholecystectomy," J. Surg. Endosc., vol. 20, pp. 135-138, 2006.

[2] M. Rentschler, J. Dumpert, S. Platt, K. Iagnemma, D. Oleynikov, and S. Farritor, "An in vivo mobile robot for surgical vision and task assistance," ASME J. Med. Devices, vol. 1, no. 1, pp. 23-29, 2007.

[3] Canadian Center for Minimal Access Surgery. [Online]. Available: http://www.cmas.ca/

[4] R. M. Satava, "Surgical robotics: The early chronicles," Surg. Laparosc. Endosc. Percutan. Tech., vol. 12, no. 1, pp. 6-16, 2002.
[5] G. H. Ballantyne, "Robotic surgery, telerobotic surgery, telepresence, and tele-mentoring," J. Surg. Endosc., vol. 16, pp. 1389-1402, 2002.

[6] M. O. Schurr, G. Buess, B. Neisius, and U. Voges, "Robotics and telemanipulation technologies for endoscopic surgery," J. Surg. Endosc., vol. 14, pp. $375-381,2000$.

[7] K. Moorthy, Y. Munz, A. Dosis, J. Hernandez, S. Martin, F. Bello, T. Rockall, and A. Darzi, "Dexterity enhancement with robotic surgery," J. Surg. Endosc., vol. 18, pp. 790-795, 2004.

[8] G. F. Dakin and M. Gagner, "Comparison of laparoscopic skills performance between standard instruments and two surgical robotic systems," J. Surg. Endosc., vol. 17, pp. 574-579, 2003.

[9] D. Nio, W. A. Bemelman, K. T. den Boer, M. S. Dunker, D. J. Gouma, and T. M. van Gulik, "Efficiency of manual vs. robotical (Zeus) assisted laparoscopic surgery in the performance of standardized tasks," J. Surg. Endosc., vol. 16, pp. 412-415, 2002

[10] W. S. Melvin, B. J. Needleman, K. R. Krause, C. Schneider, and E. C. Ellison, "Computer-enhanced vs. standard laparoscopic antireflux surgery," J. Gastrointest. Surg., vol. 6, pp. 11-16, 2002.

[11] T. Fukuda, S. Guo, K. Kosuge, F. Arai, M. Negoro, and K. Nakabayashi, "Micro active catheter system with multi degrees of freedom," in Proc. IEEE Int. Conf. Robot. Autom., San Diego, CA, 1994, pp. 2290-2295.

[12] K. Suzumori, S. Iikura, and H. Tanaka, "Development of flexible microactuator and its applications to robotics mechanisms," in Proc. IEEE Int. Conf. Robot. Autom., Sacramento, CA, 1991, pp. 1622-1627.

[13] L. Phee, D. Accoto, A. Menciassi, C. Stefanini, M. Carrozza, and P. Dario, "Analysis and development of locomotion devices for the gastrointestinal tract," IEEE Trans. Biomed. Eng., vol. 49, no. 6, pp. 613-616, Jun. 2002

[14] S. Grundfest, J. Burdick, and A. Slatkin, "The development of a robotic endoscope," in Proc. IEEE Int. Conf. Intell. Robots Syst., Nagoya, Japan, 1995 , pp. $162-171$.

[15] A. Flynn, K. Udayakumar, and D. Barret, "Tomorrow's surgery; micromotors and microrobots for minimally invasive procedures," Minim. Invasive Surg. Allied Technol., vol. 7, no. 4, pp. 343-352, 1995.

[16] P. Breedveld, E. Danielle, and M. Van Gorp, "Locomotion through the intestine by means of rolling stents," presented at the ASME Des. Eng. Tech. Conf., Salt Lake City, UT, 2004.

[17] A. Glukhovsky and H. Jacob, "The development and application of wireless capsule endoscopy," Int.J. Med.Robot. Comput. Assist. Surg., vol. 1, pp. 114-123, 2004.

[18] G. Meron, "The development of the swallowable video capsule," Gastrointest. Endosc., vol. 6, pp. 817-819, 2000.

[19] N. Patronik, M. A. Zenati, and C. Riviere, "Preliminary evaluation of a mobile robotic device for navigation and intervention on the beating heart," Comput. Aided Surg., vol. 10, no. 4, pp. 225-232, 2005.

[20] N. Patronik, M. A. Zenati, and C. Riviere, "Crawling on the heart: A mobile robotic device for minimally invasive cardiac interventions," in Medical Image Computing and Computer-Assisted Intervention, C. Barillot, D. R. Haynor, and P. Hellier, Eds. Berlin, Germany: Springer-Verlag, 2004, pp. 9-16.

[21] M. Rentschler, J. Dumpert, S. Platt, K. Iagnemma, D. Oleynikov, and S. Farritor, "Modeling, analysis, and experimental study of in vivo wheeled robotic mobility," IEEE Trans. Robot., vol. 22, no. 2, pp. 308-321, Apr. 2006.

[22] M. Rentschler, J. Dumpert, S. Platt, S. Farritor, and D. Oleynikov, "Natural orifice surgery with an endoluminal mobile robot," J. Surg. Endosc, vol. 21 , no. 7, pp. 1212-1215, 2007.

[23] V. E. M. Strong, N. J. Hogle, and D. L. Fowler, "Efficacy of novel robotic camera vs. a standard laparoscopic camera," Surg. Innov., vol. 12, no. 4, pp. 315-318, 2005

[24] J. Dumpert, M. Rentschler, S. Farritor, S. Platt, and D. Oleynikov, "Video quality analysis of in vivo surgical robots," IEEE Sensors J., to be published.

[25] C. Wittson, D. Afflek, and V. Johnson, "Two-way television group therapy," Ment. Hosp., vol. 12, pp. 22-23, 1961.

[26] E. Taniguchi and S. Ohashi, "Construction of a regional tele-mentoring network for endoscopic surgery in Japan," IEEE Trans. Inf. Technol. Biomed., vol. 4, no. 3, pp. 195-199, Sep. 2000.

[27] L. Panait, A. Rafiq, V. Tomulescu, C. Boanca, I. Popescu, A. Carbonell, and R. C. Merrell, "Tele-mentoring versus on-site mentoring in virtual reality-based surgical training," J. Surg. Endosc., vol. 20, pp. 113-118, 2006.

[28] N. Demartines, D. Mutter, M. Vix, J. Leroy, D. Glatz, F. Rosel, F. Harder, and J. Marescaux, "Assessment of telemedicine in surgical education and patient care," Ann. Surg., vol. 231, no. 2, pp. 282-291, 2000. 
[29] A. Faraz and S. Payandeh, "On inverse kinematics and trajectory planning for tele-laparoscopic manipulation," in Proc. Int. Conf. Robot. Autom., Detroit, MI, 1999, pp. 1734-1739.

[30] S. Butner and M. Ghodoussi, "A real-time system for tele-surgery," in Proc. Int. Conf. Distrib. Comput. Syst., Washington, DC, 2001, pp. 236243.

[31] T. Kim, P. M. Zimmerman, M. J. Wade, and C. A. Weiss, "The effect of delayed visual feedback on telerobotic surgery," J. Surg. Endosc., vol. 19, pp. 683-686, 2006.

[32] J. Marescaux, M. Smith, D. Folsher, F. Jamali, B. Malassagne, and J. Leroy, "Telerobotic laparoscopic cholecystectomy: Initial clinical experience with 25 patients," Ann. Surg., vol. 234, no. 1, pp. 1-7, 2001.

[33] J. Marescaux, J. Leroy, F. Rubino, M. Smith, M. Vix, M. Simone, and D. Mutter, "Transcontinental robot-assisted remote telesurgery: Feasibility and potential applications," Ann. Surg., vol. 235, no. 4, pp. 487-492, 2002.

[34] M. Anvari, C. McKinley, and H. Stein, "Establishment of the world's first telerobotic remote surgical service," Ann. Surg., vol. 241, no. 3, pp. 460-464, 2005.

[35] J. Boczko, S. Capello, D. Oleynikov, M. Rentschler, J. Dumpert, H. R. Patel, and J. V. Joseph, "Microrobots in urology: Video demonstration of microrobot-assisted laparoscopic prostatectomy and nephrectomy in a canine model," presented at the World Congr. Endourol., Cleveland, OH, Aug. 2006.

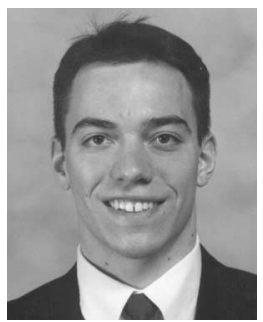

Mark E. Rentschler received the B.S. degree in mechanical engineering from the University of Nebraska, Lincoln, in 2001, the M.S. degree in mechanical engineering from the Massachusetts Institute of Technology, Cambridge, in 2003, and the $\mathrm{Ph} . \mathrm{D}$. degree in biomedical engineering from the University of Nebraska in 2006

In 2003, he was a National Defense Science and Engineering Graduate Fellow at the Massachusetts Institute of Technology. He is currently a Postdoctoral Research Associate at the University of Nebraska Medical Center, Omaha. His current research interests include the areas of surgical robotics, mechanical design, tissue mechanics, and medical mechatronics.

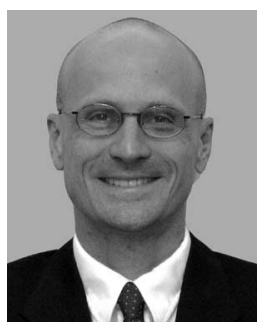

Stephen R. Platt received the B.A. degree in physics and astronomy from Williams College, Williamstown, MA, in 1983, the M.S. and Ph.D. degrees in astronomy and astrophysics from the University of Chicago, Chicago, IL, in 1991, and the M.S. degree in mechanical engineering from the University of Nebraska, Lincoln, in 2003.

$\mathrm{He}$ is currently a Research Assistant Professor in the Department of Mechanical Engineering, University of Nebraska. His current research interests include biomedical sensors, surgical robotics, and millimeter-wave detector systems for observational astrophysics applications.

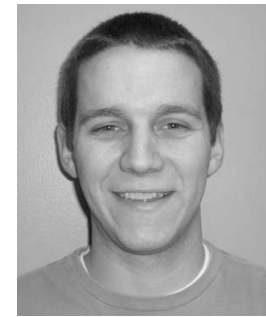

Kyle Berg received the B.S. degree in mechanical engineering in 2005 from the University of Nebraska, Lincoln, where he is currently working toward the Masters degree in mechanical engineering and is a Graduate Research Assistant.

His current research interests include abdominal wall modeling and surgical robotics.

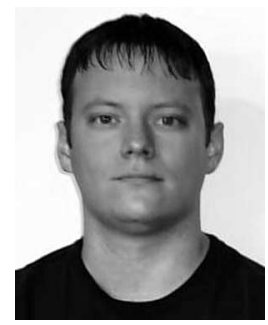

Jason Dumpert received the B.S. and M.S. degrees in electrical engineering in 2001 and 2004, respectively, both from the University of Nebraska, Lincoln, where he is currently working toward the $\mathrm{Ph} . \mathrm{D}$. degree in biomedical engineering and is a Graduate Research Assistant.

His current research interests include mobile robotics and surgical robotics.

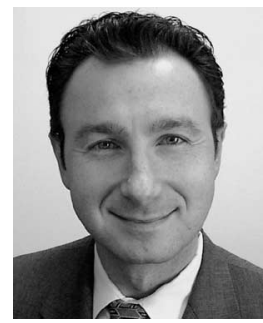

Dmitry Oleynikov received the M.D. degree from Albert Einstein School of Medicine at Yeshiva University, NY.

$\mathrm{He}$ is Board Certified in General Surgery and is an Associate Professor and Director of the Education and Training Center for Minimally Invasive and Computer-Assisted Surgery at the University of Nebraska Medical Center, Omaha. He is also an Adjunct Assistant Professor of Engineering at the University of Nebraska, Lincoln.

$\mathrm{He}$ completed the surgical residency at the University of Utah Medical Center, Salt Lake City, in 2000. After residency, he served as Acting Instructor and Senior Fellow at the Center for Videoendoscopic Surgery, Department of Surgery, University of Washington School of Medicine, Seattle. His current research interests include surgical simulation and robotics.

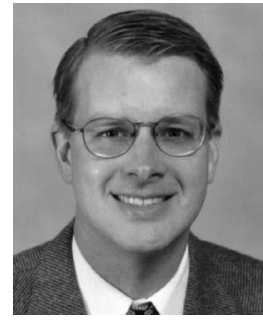

Shane M. Farritor received the B.S. degree from the University of Nebraska, Lincoln, in 1992, and the M.S. and Ph.D. degrees in mechanical engineering from the Massachusetts Institute of Technology, Cambridge, in 1998.

$\mathrm{He}$ is currently an Associate Professor of Mechanical Engineering at the University of Nebraska, and holds courtesy appointments in both the Department of Surgery and the Department of Orthopedic Surgery at the University of Nebraska Medical Center, Omaha. His current research interests include space robotics, surgical robotics, biomedical sensors, and robotics for highway safety.

Dr. Farritor serves as the Chairman of the American Institute of Aeronautics and Astronautics (AIAA) Space Robotics and Automation Technical Committee. 